\title{
ON SPACES OF TYPE $A(K)$ AND THEIR DUALS
}

\section{H. ELTON LACEY AND PETER D. MORRIS}

Let $K$ be a compact Choquet simplex and $A(K)$ the space of all continuous real-valued affine functions on $K$. Then $A(K)$ is a partially ordered Banach space with the Riesz Decomposition Property and the dual $A(K)^{*}$ of $A(K)$ is an $L$-space [15]. In particular, if $X$ is a compact Hausdorff space, then $C(X)=A(K)$ where $K$ is the compact convex set of probability measures on $X$ in the weak*-topology [2]. In this paper certain relations between $K, A(K)$ and $A(K)^{*}$ are studied. For example under certain conditions on $K, A(K)^{*}$ is a space of the type $l^{1}(D)$ if and only if the set $K_{e}$ of extreme points of $K$ contains no nonempty compact perfect subsets. The results presented here generalize some of the results in [6] and [13]. A sufficient condition for $A(K)$ to have the Dieudonné property is also presented.

1. The dual space to $A(K)$. In this section necessary and sufficient conditions for the dual of $A(K)$ to be a space of the type $l^{1}(D)$ for some set $D$ are given. Certain operator properties are used in these characterizations and for convenience they are introduced here. A continuous linear operator from a Banach space $A$ to a Banach space $B$ is said to be completely continuous if it carries weakly Cauchy sequences in $A$ into norm convergent sequences in $B$. A Banach space $A$ is said to have the Dunford-Pettis property (D.P. property) if for each Banach space $B$, each weakly compact linear operator from $A$ to $B$ is completely continuous. Reciprocally, a Banach space $A$ is said to have the R.D.P. property if for each Banach space $B$, each completely continuous linear operator from $A$ to $B$ is weakly compact. A Banach space $A$ is said to have the Dieudonné property (D. property) if for each Banach space $B$, each continuous linear operator from $A$ to $B$ which carries weakly Cauchy sequences in $A$ to weakly convergent sequences in $B$ is weakly compact. Clearly the D. property implies the R.D.P. property. Also, $A$ has the D.P. property when $A^{*}$ is an $L$-space [4, p. 136].

In the following theorem $A$ denotes a Banach space whose dual is an $L$-space. From [11, Theorem 6.1] and [15] it follows that such a space $A$ is representable as a space $A(K)$ with $K$ a compact Choquet simplex if and only if the unit sphere of $A$ contains an extreme point. 1969.

Received by the editors November 13, 1968 and, in revised form, January 29, 
1.1. ThEOREM. The following statements are equivalent.

1. $A^{*}$ is linearly isometric to $l^{1}(D)$ for some set $D$.

2. $A^{* *}$ is linearly isometric to $m(S)$ for some set $S$.

3. $A^{*}$ contains no subspace linearly isomorphic to $L^{1}[0,1]=L^{1}$.

4. $l^{2}$ (separable Hilbert space) is not a continuous linear image of $A$.

5. For each Banach space $B$, each weakly compact linear operator from $A$ to $B$ is compact.

6. If $B$ is a closed linear subspace of $A$ and $B^{*}$ is an L-space, then $B^{*}$ is linearly isometric to $l^{1}(T)$ for some set $T$.

7. If $B$ is a separable closed infinite dimensional linear subspace of $A$ and $B^{*}$ is an L-space, then $B^{*}$ is linearly isometric to $l^{1}$.

8. If $B$ is a separable closed linear subspace of $A$, then $B^{*}$ is separable.

9. For each Banach space $B$, each completely continuous linear operator from $A$ to $B$ is compact.

Proof. (1) implies (2). If $A^{*}=l^{1}(D)$ for some set $D$, then $A^{* *}$ $=m(D)$.

(2) implies (1). Suppose $A^{* *}=m(S)$ for some set $S$. By a theorem of Grothendieck [4], a dual $M$-space is the dual of a unique $L$-space. Thus $A^{*}=l^{1}(S)$.

It is well known (see e.g. [16]) that an $L$-space contains a copy of $L^{1}$ if and only if it is not of the type $l^{1}(D)$. Hence (1) and (3) are equivalent.

(not 3) implies (not 4). If $A^{*}$ contains a copy of $L^{1}$, then $A^{*}$ contains a subspace linearly isomorphic to $l^{2}$ since $l^{2}$ is linearly isomorphic to a subspace of $L^{1}$ (see e.g. [6]). If $T$ is a linear isomorphism of $l^{2}$ into $A^{*}$, then $T^{*}$ restricted to $A$ is onto $l^{2}$ since $\left(T^{*} \mid A\right)^{*}=T$ and $T$ has a bounded inverse.

(1) implies (5). Suppose $T: A \rightarrow B$ is weakly compact. Then $T^{*}$ is weakly compact. Since norm and weak convergence of sequences in $l^{1}(D)$ coincide, $T^{*}$ is compact and, thus, $T$ is compact.

(5) implies (4). Since any continuous linear operator with range space $l^{2}$ is weakly compact, this is immediate.

It has now been shown that (1)-(5) are equivalent.

(4) implies (6). Suppose $B$ is a closed linear subspace of $A$ and $B^{*}$ is an $L$-space not of the type $l^{1}(T)$. Then by (not 4$) \Rightarrow($ not 1$)$ applied to the Banach space $B$ there is a continuous linear map $T$ of $B$ onto $l^{2}$. By [11, Theorem 6.1] there is a continuous linear extension of $T$ to $A$ which contradicts (4). Thus, $B^{*}$ is of the type $l^{1}(T)$ for some set $T$.

(6) implies (7). Let $B$ be a separable closed infinite dimensional linear subspace of $A$ with $B^{*}$ an $L$-space. Then $B^{*}=l^{1}(T)$ for some set $T$. Suppose card $T>\boldsymbol{\aleph}_{0}$. Then by [12, Proposition 3.2] $B$ contains 
a subspace isomorphic to $l^{1}$. Thus according to [12, Proposition 3.3] $B^{*}$ contains a subspace isomorphic to $C(X)^{*}$ where $X$ is the Cantor set. Since $C(X)^{*}$ contains a copy of $L^{1}$ (see [13]), this is a contradiction. Hence card $T=\boldsymbol{N}_{0}$.

(7) implies (8). Let $B$ be a separable closed linear subspace of $A$. Then by $\left[11\right.$, p. 67] there is a separable closed linear subspace $B_{1}$ of $A$ such that $B_{1}$ contains $B$ and $B_{1}^{*}$ is an $L$-space. Hence, $B_{1}^{*}=l^{1}$ is separable and so is $B^{*}$.

(8) implies (9). This is shown in [8].

(9) implies (4). Suppose $T: A \rightarrow l^{2}$ is a continuous linear operator. Then $T$ is weakly compact and since $A$ has the D.P. property, $T$ is completely continuous. Hence by (9) $T$ is compact and cannot be onto $l^{2}$.

Let $K$ be a compact Choquet simplex. If the set $K e$ of extreme points of $K$ is a Borel set and for each maximal probability measure $\mu$ on $K, \mu(K e)=1, K$ is said to be maximally supported. The simplex $K$ is said to be an $r$-simplex if $K e$ is closed. In such a case $A(K)=C(K e)$.

The following theorem explicitly reveals the $L$-space structure of $A(K)$ and is useful in establishing some of the results presented later on. Its proof is easily checked.

1.2. Theorem. Let $K$ be a compact Choquet simplex, $X$ the closure of the extreme points of $K, A$ the set of restrictions of the elements of $A(K)$ to $X, Q$ the set of restrictions of the nonnegative maximal measures on $K$ to $X$, and $M=Q-Q\left(\right.$ in $\left.C(X)^{*}\right)$. Then $M$ is a closed linear subspace of $C(X)^{*}, C(X)^{*}=M \oplus A^{\perp}$, the quotient map $\pi: C(X)^{*} \rightarrow C(X)^{*} / A^{\perp}$ is an order isomorphism and, when restricted to $M$, a linear isometry. In particular, $A^{*}$ is order isomorphic and linearly isometric to $M$.

REMARKs. That the restriction map $h \rightarrow h \mid X$ is an order isomorphism and a linear isometry is well known. The space $C(X)^{*} / A^{\perp}$ has the quotient norm and the natural order induced by the natural map of $A^{*}$ onto $C(X)^{*} / A^{\perp} . A^{\perp}$ is the annihilator of $A$ in $C(X)^{*}$. Maximal measures are defined, for example, in $[14$, p. 25].

It is well known that if $\mu \in Q$, then $\mu(\{x\})=0$ for all $x \in K \backslash K e$ (see [2]). Thus the only purely atomic measures that are maximal are the ones whose atoms lie in $K e$. Conversely, every purely atomic measure whose atoms lie in $K e$ is maximal $\left[14\right.$, p. 70]. If $l^{1}(K e)$ denotes the sublattice of $C(X)^{*}$ of purely atomic elements whose atoms lie in $K e$, then $l^{1}(K e)$ consists precisely of the purely atomic elements of $M$.

1.3. Lemma. Let $F \subseteq K e$ be a compact set. Then $K_{0}=[c o]^{-}(F)$ is a closed face in $K$, and, in fact, an r-simplex. 
Proof. Let $x \in K_{0}, y, z \in K$ with $x=t y+(1-t) z$ for some $0<t<1$. Let $\mu, \nu$ be maximal probability measures representing $y, z$ respectively. Since $x \in K_{0}$ and $\left(K_{0}\right) e=F$ is closed, the maximal probability measure $\sigma$ representing $x$ is supported on $F$. Hence $\mu$ and $\nu$ are supported on $F$ and $y, z$ are in $K_{0}$ (see [14, Proposition 1.2]). Thus $K_{0}$ is a face of $K$.

Now, suppose $\mu, \nu$ are two maximal probability measures on $K_{0}$ representing the same point. Then $\mu$ and $\nu$ are maximal in $K[14$, p. 70] and represent the same point in $K$. Hence $\mu=\nu$ and $K_{0}$ is a simplex. Since $\left(K_{0}\right) e$ is closed, $K_{0}$ is an $r$-simplex.

1.4. TheOREM. The following statements are equivalent.

1. $A(K)^{*}$ is linearly isometric to $l^{1}(D)$ for some set $D$.

2. $A(K)^{*}$ is linearly isometric to $l^{1}(K e)$.

3. $K$ admits no maximal nonatomic measure.

4. $A(K)^{* *}$ is linearly isometric to $m(S)$ for some set $S$.

5. $A(K)^{* *}$ is linearly isometric to $m(K e)$.

6. $A(K)$ contains no infinite dimensional reflexive subspace.

If $K$ is maximally supported, then 1-6 are equivalent to

7. Ke contains no nonempty perfect compact subsets.

Proof. By Theorem $1.2 A(K)^{*}=M=l^{1}(K e) \oplus N$ where $N$ is the linear span of the maximal nonatomic measures. Moreover, if $\mu$ is a maximal nonatomic measure, then $L^{1}(\mu) \subseteq N$. Since for $0 \neq \mu \in N$, $L^{1}(\mu)$ contains a copy of $L^{1}[0,1], N \neq\{0\}$ implies $N$ contains a copy of $L^{1}$.

(1) implies (2). By the above, $A(K)^{*}=l^{1}(D)$ implies $N=\{0\}$. Hence $A(K)^{*}=l^{1}(K e)$.

(2) is equivalent to (3). $A(K)^{*}=l^{1}(K e)$ if and only if $N=\{0\}$.

(2) implies (1). Clear.

By Theorem 1.1, (2), (4) and (5) are equivalent. Thus they are all equivalent to any one of the nine statements in Theorem 1.1 also.

(1) implies (6). Suppose $A(K)$ contains an infinite dimensional reflexive subspace. Let $B$ be a separable infinite dimensional reflexive subspace of $A$ and $B_{1}$ a separable subspace of $A$ containing $B$ such that $B_{1}^{*}$ is an $L$-space $\left[11\right.$, p. 67]. By Theorem $1.1(7), B_{1}^{*}=l^{1}$. In particular the set of extreme points of the unit sphere of $B_{1}^{*}$ is countable. If $J$ is the natural map of $B$ into $B_{1}$, then the adjoint $J^{*}$ of $J$ carries the unit sphere of $B_{1}^{*}$ onto the unit sphere of $B^{*}$. Hence the set of extreme points of the unit sphere of $B^{*}$ is countable. But, the set of extreme points of the unit sphere of an infinite dimensional reflexive space is uncountable [10, Theorem 1.1].

(not 1) implies (not 6). If $A(K)^{*}$ is not of the type $l^{1}(D)$ for some 
set $D$, then by Theorem 1.1(7) there is a separable closed linear subspace $B$ of $A(K)$ containing the constant function 1 such that $B^{*}$ is an $L$-space and $B^{*} \neq l^{1}$. Now, the argument in (6) implies (7) of the proof of Theorem 1.1 shows that $B^{*}$ is not of the type $l^{1}(T)$ for any set $T$. Moreover $B=A(L)$ where $L=\left\{\mu \in B^{*}: \mu(1)=1=\|\mu\|\right\}$ and $L$ is a compact Choquet simplex in the weak*-topology (see, e.g. [15]). Since $B$ is separable, $L$ is metrizable. Thus $L e$ is a $G_{\delta}$-set and $L$ is maximally supported $\left[14\right.$, p. 19]. Since $A(L)^{*}$ is not of the type $l^{1}(T), L$ admits a maximal nonatomic measure $\mu$. Since $\mu(L e)=1$, there is a nonempty perfect compact set $F \subseteq L e$. By Lemma 1.3 $L_{0}=[c o]^{-}(F)$ is a closed face in $K$ and an $r$-simplex and by [9, Theorem 3.5] there is an affine continuous map $h: L \rightarrow L_{0}$ such that $L(x)=x$ for all $x \in L_{0}$. Hence the operator $T(f)=f \circ h$ is a linear isometric embedding of $A\left(L_{0}\right)$ into $A(L)$. But, $A\left(L_{0}\right)=C(F)$ and $C(F)$ contains copies of all separable Banach spaces (see e.g. [7]). Hence $A(K)$ contains copies of all separable reflexive spaces.

(not 7) implies (not 3). If $K e$ contains a perfect set $F$ and $\mu$ is a nonatomic probability measure on $K$ such that $\mu(F)=1$, then $\mu$ is maximal since it is supported on $K e[14$, p. 70].

(not 3 ) implies (not 7). If $\mu$ is a maximal nonatomic probability measure on $K$, then since $K$ is maximally supported, $\mu(K e)=1$. Hence there is, for example, a perfect compact set $F \subseteq K e$ such that $\mu(F)=\frac{1}{2}$.

REMARK. Of course, if $[\mathrm{Ke}]^{-}$is dispersed, then $\mathrm{Ke}$ contains no nonempty perfect compact subsets. However, it is possible to have a metrizable simplex $K$ with $K e$ countable and $[K e]^{-}$uncountable.

2. Some convergence properties in $A(K)^{*}$. In this section we give a sufficient condition for $A(K)$ to have the Dieudonné property. For a compact Choquet simplex $K$, let $B_{1}$ denote the first Baire class of bounded functions on $K$, i.e., the elements of $B_{1}$ are point-wise limits of uniformly bounded sequences of continuous functions on $K$. Let $A_{1}, A_{2}$ denote the first and second affine Baire classes of bounded functions on $K$, i.e., the elements of $A_{1}$ (resp. $\left.A_{2}\right)$ are point-wise limits of uniformly bounded sequences of elements of $A(K)$ (resp. $A_{1}$ ). The Dieudonné property can be stated as follows: $A(K)$ has the D. property if and only if each bounded convex circled set in $A(K)^{*}$ which is compact relative to the weak topology $\sigma\left(A(K)^{*}, A_{1}\right)$ is also compact relative to the weak topology $\sigma\left(A(K)^{*}, A(K)^{* *}\right)$. In particular, $C(K)$ has the D. property.

For each bounded Borel function $f$ on $K$ let $(P f)(x)=\int f d \mu_{x}$, where for each $x \in K, \mu_{x}$ is the unique maximal probability measure which represents $x$. 
2.1. LemmA. Let $K$ be a simplex and suppose that $K e$ is a countable union of compact sets. Let $\left\{f_{n}\right\}$ be a uniformly bounded sequence of continuous functions on $K$ that converge point-wise to a function $g$ on $K$. Then there is a sequence $\left\{h_{n}\right\}$ of uniformly bounded affine continuous functions on $K$ converging point-wise to $P(g)$.

Proof. Let $F_{1} \subseteq F_{2} \subseteq \cdots$ be a sequence of compact sets such that $\bigcup_{n=1}^{\infty} F_{n}=K e$. By $[9$, Theorem 3.3] and Lemma 1.3 there is, for each $n$, an $h_{n} \in A(K)$ such that $h_{n}\left|F_{n}=f_{n}\right| F_{n}$ and $\left\|h_{n}\right\|=\left\|f_{n} \mid F_{n}\right\| \leqq\left\|f_{n}\right\|$ $\left(f_{n} \mid F_{n}\right.$ clearly has a continuous affine extension to $K_{n}=[c o]-\left(F_{n}\right)$ since $K_{n}$ is an $r$-simplex, see [2]). Clearly $h_{n}(x) \rightarrow g(x)$ for all $x \in K e$. For each $x \in K$ let $\mu_{x}$ denote the unique maximal probability measure on $K$ which represents $x$. If $h(x)$ is defined to be $\int g d \mu_{x}$, then $h$ is affine and by the dominated convergence theorem, $h_{n}(x)=\int h_{n} d \mu_{x} \rightarrow \int g d \mu_{x}$ $=h(x)$ for each $x \in K$ (recall that $K$ is maximally supported so that $\mu_{x}(K e)=1$ for all $x \in K,[14$, p. 70]).

2.2. ThEOREM. If $K e$ is a countable union of compact sets, then $A(K)$ has the Dieudonne property.

Proof. Let $D$ be a bounded convex circled $\sigma\left(A(K)^{*}, A_{1}\right)$-compact set in $A(K)^{*}$. If $\left\{\mu_{d}\right\}$ is a net in $D$, then there is a subnet $\left\{\nu_{a}\right\}$ of $\left\{\mu_{d}\right\}$ that converges to some element $\mu \in D$. Let $f \in B_{1}$. Then $P(f) \in A_{1}$ and since $K$ is maximally supported and $P(f)=f$ on $K e, \int f d \nu=\int P f d \nu$ for each $\nu \in A(K)^{*}=M$. Thus $\nu_{a}(P f) \rightarrow \mu(P f)$ implies $\nu_{a}(f) \rightarrow \mu(f)$ and $\left\{\nu_{a}\right\}$ converges to $\mu$ relative to the $\sigma\left(C(K)^{*}, B_{1}\right)$ topology. Since $C(K)$ has the D. property, $D$ is compact in the $\sigma\left(C(K)^{*}, C(K)^{* *}\right)$ topology and hence in the $\sigma\left(A(K)^{*}, A(K)^{* *}\right)$ topology.

REMARK. The above proof actually establishes that if $K$ is maximally supported and if for each $f \in B_{1}, P(f) \in A_{1}$, then $A(K)$ has the D. property. However the only case where we know that this occurs is the one where $K e$ is a countable union of compact sets. If we use $A_{2}$ instead of $A_{1}$, we get the following result.

2.3. Theorem. If $K$ is a metrizable compact Choquet simplex, then each bounded convex circled set $D$ in $A(K)^{*}$ which is compact in the $\sigma\left(A(K)^{*}, A_{2}\right)$ topology is compact in the $\sigma\left(A(K)^{*}, A(K)^{* *}\right)$ topology.

Proof. It suffices to show that $D$ is compact in the

$$
\sigma\left(C(K)^{*}, C(K)^{* *}\right)
$$

topology. Since $C(K)$ is separable, it suffices to show that for each sequence $\left\{\mu_{n}\right\}$ in $D$ there is a subsequence $\left\{\nu_{n}\right\}$ of $\left\{\mu_{n}\right\}$ and an element $\mu$ in $D$ such that for each open set $U$ in $K, \nu_{n}(U) \rightarrow \mu(U)$ [3]. 
Since $A(K)$ is separable and the $\sigma\left(A(K)^{*}, A(K)\right)$ and $\sigma\left(A(K)^{*}, A_{2}\right)$ topologies agree on $D, D$ is metrizable in the $\sigma\left(A(K)^{*}, A_{2}\right)$ topology. Let $\left\{\mu_{n}\right\}$ be a sequence in $D$. Since $D$ is compact and metrizable there is a subsequence $\left\{\nu_{n}\right\}$ of $\left\{\mu_{n}\right\}$ which converges relative to $A_{2}$ to some element $\mu$ in $D$. Let $U$ be an open set in $K$ and $f$ the characteristic function of $U$. Since $f$ is upper semicontinuous, $P(f) \in A_{2}$ [1, Proposition 4]. Since $K$ is maximally supported and $P(f)=f$ on $K e, \nu(f)$ $=\nu(P f)$ for all $\nu \in A(K)^{*}$. Now, $\nu_{n}(P f) \rightarrow \mu(P f)$ which implies $\nu_{n}(f)$ $\rightarrow \mu(f)$. But $\nu_{n}(U)=\nu_{n}(f) \rightarrow \mu(f)=\mu(U)$.

\section{REFERENCES}

1. E. Alfsen, $A$ note on the Borel structure of a metrizable Choquet simplex and of its extreme boundary, Math. Scand. 19 (1966), 161-171.

2. Heinz Bauer, Konvexität in topologischen Vektorräumen, Lecture notes, University of Hamburg, 1963/64.

3. J. Dieudonné, Sur la convergence des suites de mesures de Radon, An. Acad. Brasil. Ci. 23 (1951), 21-38.

4. A. Grothendieck, Sur les applications linéaires faiblement compactes d'espaces $d u$ type $C(K)$, Canad. J. Math. 5 (1953), 129-173.

5. - Une caractérisation vectorielle-métrique des espaces $L^{1}$, Canad. J. Math. 7 (1955), 552-561.

6. H. E. Lacey and P. D. Morris, Continuous linear operators on spaces of continuous functions, Proc. Amer. Math. Soc. 17 (1966), 848-853.

7. - On universal spaces of the type $C(X)$, Proc. Amer. Math. Soc. 19 (1968), $350-353$.

8. H. E. Lacey and R. J. Whitley, Conditions under which all the bounded linear operators are compact, Math. Ann. 158 (1965), 1-5.

9. A. J. Lazar, Spaces of affine continuous functions, Trans. Amer. Math. Soc. 134 (1968), 503-525.

10. J. Lindenstrauss and R. R. Phelps, Extreme point properties of convex bodies in reflexive Banach spaces, Israel J. Math. 6 (1968), 39-48.

11. J. Lindenstrauss, Extensions of compact operators, Mem. Amer. Math. Soc. No. 48, (1964), 112 pp.

12. A. Pelcžyński, On Banach spaces containing $L_{1}(\mu)$, Studia Math. 30 (1968), 231-246.

13. A. Pelcžyński and Z. Semadeni, Spaces of continuous functions. III, Studia Math. 18 (1959), 211-222.

14. R. R. Phelps, Lectures on Choquet's theorem, Van Nostrand, New York, 1966.

15. Z. Semadeni, Free compact convex sets, Bull. Acad. Polon. Sci. Sér. Sci. Math. Astronom. Phys. 13 (1965), 141-146.

16. - "Spaces of continuous functions" in Advances in mathematics. Vol. 1, Academic Press, New York, 1965, pp. 319-382.

University of TeXas at Austin and Pennsylvania State University 